\title{
Infections in burned children: epidemiological and risk factor analysis
}

\author{
María Teresa Rosanova, M.D., ${ }^{a}$ Daniel Stamboulian, M.D., ${ }^{b}$ and Roberto Lede, M.D. ${ }^{c}$
}

\begin{abstract}
Introduction. Burns are the third cause of accidentaldeathsamong children. Approximately $50-60 \%$ of these deaths are the result of an infection.

Objective. To determine infection related risk factors in burned children.

Population and Methods. All patients admitted to the Burn Unit of Hospital "Prof. Dr. Juan P. Garrahan" between June 2007 and December 2009 were included. The epidemiology of hospital-acquired infections and the associated

infection, understudied in children, would enable the implementation of measures aimed at reducing their incidence. ${ }^{1}$

Our main objective was to assess risk factors of hospital-acquired infections in burned children and to determine the epidemiology of these infections in this population.
\end{abstract} outcome measures were determined. Groups of infected and non-infected children were compared using Student's t test or the MannWhitney Rank Sum test, as applicable.

Dichotomous outcome measures were analyzed with the X2 test using Yates' correction. In order to assess the predictive value of independent outcome measures, the multiple logistic regression model was applied.

Results. In this cohort of 110 children, 128 hospital-acquired infections were recorded in 84 patients. There were 17 deaths $(15 \%) ; 14$ out of these $17(82 \%)$ were related to infection. Infection-related factors included the percentage of burned body surface area; the highest Garces' index; burn depth; antibiotic prophylaxis; the use of topical antibiotics; the presence of a central venous line, an arterial line, a urinary catheter, mechanical ventilation support, escharotomy, and the need of a graft. The multivariate analysis showed a higher risk of infection with the use of central venous lines (RR: 5.15; 95\% CI: 1.4418.46), antibiotic prophylaxis (RR: $5.22 ; 95 \%$ CI: 1.26-21.63), and graft requirement (RR:3.65;95\% CI: 1.08-12.37).

Conclusions. The presence of lines or catheters, antibiotic prophylaxis, and graft requirement were independent risk factors for infection in burned children.

Key words: burned, infections, children.

http:/ /dx.doi.org/10.5546/aap.2013.303

\section{INTRODUCTION}

According to the Argentine Burn Association, the annual incidence of burns in Argentina is 5/1000, and accounts for the third cause of they account death among children. ${ }^{1}$

Infection leads to death in 50$60 \%$ of burned patients. ${ }^{1-3}$ The identification of risk factors for

\section{POPULATION AND METHODS}

Prospective cohort of all burned pediatric patients admitted to the Hospital "Prof. Dr. Juan P. Garrahan" between June 2007 and December 2009.

Children aged from 1 month to 18 years old admitted to the burn unit due to any degree burns with one week evolution were included.

Patients with infection at the time of admission were excluded.

Independent outcome measures were: age in months; sex; type of burn: superficial or $A$, intermediate or $A B$, and deep or B; cause of burn (fire, hot liquids, electrical, others); burned surface area expressed as a percentage of the body surface area; history of event related hospitalization, including days, type and duration of prophylactic antibiotic therapy used at the time of admission to this hospital, and type of treatment (surgical, topical or systemic antimicrobial therapy); use and length of balneotherapy; use of mechanical ventilation support; central or peripheral lines; parenteral nutrition, and length of treatment with each of these procedures.

The primary dependent outcome measure was the presence of a hospital-acquired infection. A hospital-acquired infection was defined as an infection developed in the 48 hour period following the admission to a hospital and not 
present at the time of admission.

The secondary outcome measures were:

a. Type of microorganism.

b. Length of treatment in days.

c. Progress. In case of death, progress was considered related to the infection if death occurred in the presence of positive cultures or clinical signs of infection.

d. Length of hospital stay.

\section{Definitions}

Garces' index: ${ }^{1-3}$ this a severity and mortality prediction index calculated as follows: 40 minus patient's age, plus burn percentage, multiplied times 1 (if type A burn), times 2 (if type AB burn), or times 3 (if type B burn).

From 0 to 60 points: grade 1 (mild risk).

From 61 to 90 points: grade 2 (moderate risk).

From 91 to 120 points: grade 3 (severe risk).

Over 121 points: grade 4 (critical risk).

Inhalation syndrome: suspected: if the patient exposed to smoke or heat had a nasal or mouth burn, carbonaceous sputum, dysphonia, cough, rales, nasopharyngeal erythema or edema, or carbon monoxide poisoning; certain: if the patient's fibrobronchoscopy shows a lesion in the respiratory tract mucous membrane.

Type of infections: according to the American Burn Association (ABA), 4 the following types of infections have been defined: a) burn wound infection: if there were local signs of infection or a positive microbiological culture in viable tissue samples; $b$ ) sepsis associated with a burn wound: if there was evidence of a wound infection and concurrent organ failure; c) line or catheterassociated infection: presence of positive blood culture in patients with a central line with no other apparent cause of infection.

\section{Statistical Analysis}

The SPSS software for Windows, version 11.5, was used. Logistic regression was performed applying the forward stepwise method. In order to assess differences between groups in terms of continuous outcome measures, Student's t test or the Mann-Whitney Rank Sum test was used, as applicable, with the outcome distribution.

The X2 test was used for dichotomous outcome measures (with Yates' correction). For the assessment of the predictive value of independent outcome measures, a multiple logistic regression model integrating outcomes that showed an association with a $<20 \%$ confidence interval to the specified endpoint was used. The power of the association between outcome measures was expressed as relative risk (RR).

\section{RESULTS}

A hundred and ten patients participated, and their clinical and epidemiological characteristics are summarized in Table 1 . In relation to invasive procedures, 90 patients ( $82 \%$ ) had a venous line, with a mean dwell time of 16.5 days (r: 1-90); 83 patients (75.5\%) had an arterial line (mean: 16 days; r: $1-87$ days), and 86 patients (78\%) had a urinary catheter (mean: 16 days; r: 1-80). Only 1 patient $(1 \%)$ required parenteral feeding during 7 days. A total of 75 patients $(68 \%)$ required mechanical ventilation, with a mean use of 14 days (r: 1-180). Balneotherapy was used in 93 patients $(85 \%)$ during 7 days (mean: 7 days; r: 1-44). Topical antibiotics were prescribed in 95 patients $(86 \%)$, while $92(84 \%)$ received systemic antibiotics, either as prophylaxis or treatment. Colistin was used in 68 children (62\%).

In total, 128 hospital-acquired infections were recorded in 84 patients; the most common clinical presentation was burn-related sepsis. Bacterial infections accounted for the most frequent type of infections, the multidrug-resistant Gram-negative bacteria, Pseudomonas aeruginosa and Acinetobacter spp., were the most commonly isolated bacteria. The (mean) time until development was 10 days (r: 2-84). The most common fungal infections were caused by Candida albicans and no albicans, and in this case, the (mean) time until development was 10.5 days (r: 2-60). S. aureus was the most common Gram-positive bacteria (mean time until isolation: 6.5 days; r: 2-56) (see Table 2).

A baseline escharotomy was performed in 90 patients (82\%; mean: 5 days; r: $1-19)$. A total escharotomy was completed at 5 days (r: $1-45)$. Eighty-two patients (74.5\%) required a graft, with a mean of 17 days (r: 1-81).The median hospital length of stay was 37 days (r: 1-139).

A total of 93 patients (85\%) were discharged, and 17 patients (15\%) died. The cause of death was related to the infection in 14 of these cases $(82 \%)$.

A bivariate analysis of infected and noninfected patients showed no significant differences between both groups in terms of age $(\mathrm{p}=0.079)$, distribution by $\operatorname{sex}(\mathrm{p}=0.131)$, burn etiology (mechanism) $(\mathrm{p}=0.216)$, location of the burn injury $(\mathrm{p}=0.07)$, or the presence of an inhalation syndrome $(p=0.139)$.

The percentage of burned body surface area, a higher Garces' index, and the type of burn 
(depth) showed a significant association with the development of an infection; type B burns had the highest risk of infection.

Antibiotic prophylaxis was observed to be related to the development of an infection, as well as the use of topical antibiotics, the presence of a central venous line, an arterial line, a urinary catheter, mechanical ventilation, an escharotomy, and graft requirement. Significant differences were found in the number of days until a complete graft placement between the patients with and without infection $(\mathrm{p}=0.026)$.

The non-infected group completed the graft placement on day 10 , in average, versus 22.5 days in the infected group. The mean difference was 12 days (95\% CI: 5-20). Balneotherapy was associated with a higher risk of infection (see Table 3).

\section{Multivariate Analysis}

By integrating all significant outcome measures used in the bivariate analysis, it was possible to establish that the use of central venous lines, antibiotic prophylaxis, and graft requirement were independent risk factors for infection (see Table 4).

\section{DISCUSSION}

Infections in burned patients are a determining factor of morbidity and mortality; therefore, it is important to know which factors are associated with the development of infections in children, a population in whom studies are scarce.

Patients' age has been suggested as a risk factor of infection in children, but as commented by Rodgers, et al., ${ }^{2}$ in our study, patients' age did not play this role. Although men had a higher risk of infection in a series of adult patients, this was not observed in our study. ${ }^{5}$

The risk of infection is higher when the burned body surface area is over $30 \%$ due to the associated immune complications, ${ }^{2-6}$ but it was not an independently associated factor in this series.

Patients with a higher Garces' index were more prone to infection. Garces' index considers, among other parameters, age, surface and depth of burn; therefore, by including these outcome

TABLE 1. Characteristics of patients ( $n: 110)$

\begin{tabular}{|c|c|c|c|}
\hline Outcome measure & $n$ (patients) & Percentage & Median \\
\hline Age & & & $\begin{array}{l}31.5 \text { months old } \\
\text { (r: } 1-24 \text { months old) }\end{array}$ \\
\hline Male & 71 & 66 & \\
\hline Underlying disease & 6 & 5 & \\
\hline Burn mechanism & $\begin{array}{c}\text { Fire: } 43 \\
\text { Liquid: } 31 \\
\text { Others: } 36\end{array}$ & $\begin{array}{l}39 \\
28 \\
33\end{array}$ & \\
\hline Burned surface area & & & $\begin{array}{c}27 \% \\
\text { (r: } 1-95 \%)\end{array}$ \\
\hline \multicolumn{4}{|l|}{ Garces' index } \\
\hline $\begin{array}{l}1 \\
2 \\
3 \\
4\end{array}$ & $\begin{array}{l}14 \\
39 \\
24 \\
33\end{array}$ & $\begin{array}{l}13 \\
35 \\
22 \\
30\end{array}$ & \\
\hline \multicolumn{4}{|l|}{ Type of burn } \\
\hline $\begin{array}{l}\mathrm{A} \\
\mathrm{AB} \\
\mathrm{B}\end{array}$ & $\begin{array}{l}39 \\
19 \\
52\end{array}$ & $\begin{array}{l}36 \\
17 \\
47\end{array}$ & \\
\hline Inhalation syndrome & 52 & 47 & \\
\hline Antibiotic prophylaxis & 92 & 85 & \\
\hline $\begin{array}{l}\text { Invasive procedures: } \\
\text { Venous line }\end{array}$ & 90 & 82 & \\
\hline Arterial line & 83 & 75 & \\
\hline Urinary catheter & 86 & 78 & \\
\hline MV & 75 & 68 & \\
\hline Balneotherapy & 93 & 85 & \\
\hline
\end{tabular}


measures, depth would not be a risk factor by itself.

Burns in the lower limbs or the perianal area have been considered to be more prone to infection, but as in Rodgers, et al. ${ }^{2}$ paper, in our study the location of the burn was not related to infection.

The presence of inhalation syndrome has been related to infections, especially respiratory infections. However, this is more common in adults with a higher rate of burns by fire than in children, who usually suffer hot liquid burns. This is confirmed by our study given that patients with this type of burns did not have a higher risk. ${ }^{6-10}$

Line or catheter-related infectious complications are very common. The incidence of line or catheter related infections in critical patients is variable, with an incidence rate of 2-30 episodes due to bacteremia for every 1000 days of catheter use. In this study, the presence of a line or catheter was an independent risk factor for infection and, since this could be a modifiable risk factor, prevention measures should be maximized at the time of line or catheter insertion and while the vascular access is maintained. It has been attempted to prevent infections with the use of antibiotic-impregnated lines or catheters, more frequent line or catheter replacements, a strict control of the insertion procedure, among other measures; results have been variable. ${ }^{11-14}$

Ramos, et al. ${ }^{15}$ conducted a study to evaluate the relationship between the line or catheterinsertion distance to the burned area and infection in adults. Colonization was registered more frequently in the catheter close to the burned area; so Ramos, et al. concluded that the line or catheter should not be inserted near the burned area or replacements should be more frequent.

In pediatric patients, this is complicated by the lack of venous accesses and burn extension on the child's total body surface area. A periodic replacement of IV lines has also been proposed as prevention of related bacteremias. However, the systematic rotation of lines or catheters is controversial. ${ }^{16}$ Kowalewska-Grochowska, et al. ${ }^{17}$ compared line or catheter removal on Day 7 versus line or catheter replacement using a guidewire on Day 3 and removal on Day 7, but no significant differences were observed.

Mechanical ventilation has been related to ventilator-associated pneumonia in critical patients; in our study, it was not an independent risk factor for infection. ${ }^{2,11}$

Urinary catheter is associated with the risk of urinary tract infection. Among our patients, it was not an independent risk factor for infection.

Balneotherapy can be a potential source for microorganism transmission, which could be avoided if recommendations for prevention were adequately followed. ${ }^{18}$ In this study, balneotherapy was not an independent risk factor for infection.

The escharotomy of necrotic tissue has been indicated as a protection factor against infection. Lloyd, et al. ${ }^{19}$ reported that the incidence of sepsis decreased from $6 \%$ to $1 \%$ with an early incision, and confirmed a significant reduction of mortality with an early escharotomy when compared to conventional surgical management. In these patients, escharotomy was not an independent risk factor, probably due to the higher number of necessary procedures and the possibility of bacteremia secondary to the escharotomy, or because the non-infected group had this procedure performed earlier.

One of the challenges in burn units at the time of preventing infections is the adequate use of antibiotics, whether topical or systemic..$^{20,21}$ The study conducted by Ergün, et al. ${ }^{22}$ was not able to demonstrate that systemic antibiotic prophylaxis could prevent infections. On the contrary, it was related to higher rates of infection, which is consistent with the observations of this series. Rodgers, et al. ${ }^{23}$ concluded that, in children, the use of perioperative antibiotics is not beneficial.

A graft is considered as the best definitive treatment and has proven to be useful for infection prevention. ${ }^{27-29}$ Graft requirement was an independent predictive factor for infection in our patients.

However, since this is correlated with burn depth and a greater burned body surface area, it could be concluded that these factors would be related to the fact that the graft was not a protection factor against infection or that, in average, the graft was placed later in the infected group.

Reported mortality varies between $3.5 \%$ and $7 \%$, and infections are the main cause of death in burn units. ${ }^{30}$ The high mortality rate $(15 \%)$ in these patients could be attributed to the fact that our facility is a referral center for critical patients and, among them, infection was the leading cause of death.

Although the sample size is not large enough for some of the studied outcome measures to make definitive conclusions, we believe that since this is, as far as we know, the first study 
about risk factors for infection in pediatric burned patients conducted in Argentina, these findings are greatly significant and serve as the foundation to continue gathering patients to involve a larger population. Nonetheless, this is a referral tertiary care center and data obtained could correspond to a group of patients with a more severe status and could not be extrapolated to the entire population of burned children.

\section{CONCLUSIONS}

In this study, the presence of venous lines, antibiotic prophylaxis, and graft requirement were independent risk factors for infection in burned children.

\section{Acknowledgments}

To all the members of the Plastic Surgery and Burn Unit, the Microbiology Department, and the Infectious Disease Department of Hospital "Prof. Dr. Juan P. Garrahan."

\section{REFERENCES}

1. Schwartz R, Chirino C, Saenz SV, Rodríguez T. Algunos aspectos del manejo del paciente quemado en un servicio de cirugía infantil. A propósito de 47 pacientes pediátricos. Rev Argent Dermatol 2008;89:98-103.

2. Rodgers G, Mortensen J, Fisher M, Lo A, et al. Predictors of infectious complications after burn injuries in children. Pediatr In!ect Dis J 2000;19(10):990-5.

3. Cabrera R, Pintado Otero R, Rey Calero J, García Torres V. Complicaciones infecciosas de los enfermos quemados. Cir Plás Iberolatinoam 1988;14(4):1-8.

4. Greenhalgh DG, SaffleJR,HolmesJH, Gamelli RL, etal. American Burn Association consensus conference to define sepsis and infections in burns. J Burn Care Res 2007;28(6):776-90.

5. Mayhall CG. The epidemiology of burn wound infections. Then and now. Clin Infect Dis 2003;37(4):543-50.

6. Sheridan RL. Sepsis in pediatric burn patients. Pediatr Crit Care Med 2005;6(Suppl 3):s112-9.

7. Demirdjian G, Muñoz W. Análisis estadístico de los primeros 3 años de la unidad de quemados del Hospital Garrahan. Rev Cir Infant 1997;7(1):31-5.

8. Derganc M. Present trends in fluid therapy, metabolic care, and prevention of infection in burned children. Crit Care Med 1993;21(Suppl 9):s397-9.

9. Barret JP, Ramzy PI, Wolf SE, Herndon DN. Sensitivity and specifity of bronchoalveolar lavage and protected bronchial brush in the diagnosis of pneumonia in pediatric burn patients. Arch Surg 1999;134(11):1243-7.

10. Mosier M, Pham T. American Burn Association Practice Guidelines for Prevention, diagnosis, and treatment of ventilator associated pneumonia (VAP) in burn patients. J Burn Care Res 2009;30(6): 910-28.

11. Franceschi D, Gerding R, Phillips G, Fratianne R. Risk factors associated with intravascular catheters infections in burned patients a prospective study. J Trauma 1989;29(6):811-6.

12. Kealey G, Chang P, Heinle J, Rosenquist M, Lewis RW. Prospective comparison of two management strategies of central venous catheters in burn patients. J Trauma 1995;38(3):344-9.

13. Sheridan RL, Weber JM, Peterson HF, Tompkins RG. Central venous catheter sepsis with weekly catheter change in paediatric burn patients: An analysis of 221 catheters. Burns 1995;21(2):127-9.

14. Mermel LA. Prevention of intravascular catheter-related infections. Ann intern Med 2000;132(5):391-402.

15. Ramos GE, Bolgiani AN, Patiño O, Prezzavento GE, etal. atheter infection risk related to the distance between insertion site and burned area. J Burn Care Rehabil 2002;23(4):266-71.

16. Weber J, McManus A, Nursing Committee of the International Society for Burn injuries. Infection control in burn patients. Burns 2004;30(8):A16-24.

17. Kowalewska- Grochowska K, Richards R, Moysa G, Lam $K$, et al. Guidewire catheter change in central venous catheter biofilm formation in a burn population. Chest 1991;100(4):1090-5.

18. Kluytmans J. Surgical Infections Including Burns. En: WenzelP (ed). Prevention and control of nosocomial infections. 3rd ed. Baltimore: Williams \& Wilkins; 1997. Págs.841-65.

19. Lloyd JR, Hight DW. Early laminar excision: improved control of burn wound sepsis by partial dermatome debridement. J Pediatr Surg 1978;13(6D):698-706.

20. Ramos G, Resta M, Machare Delgado E, Durlach R, et al. Systemic perioperative antibiotic prophylaxis may improve skin autograft survival in patients with acute burns. J Burn Care Res 2008;29(6):917-23.

21. Mozingo D, McManus A, Kim S, Pruitt BA Jr. Incidence of bacteremia after burn wound manipulation in the early postburn period. J Trauma 1997;42(6):1006-10.

22. Ergün O, Celik A, Ergün G, Ozok G. Prophylactic antibiotic use in pediatric burn units. Eur J Pediatr Surg 2004;14(6):422-6.

23. Rodgers GL, Fisher MC, Lo A, Cresswell A, Long SS. Study of antibiotic prophylaxis during burn wound debridement in children. J Burn Care Rehabil 1997;18(4):342-6.

24. Rosanova M, Epelbaum C, Norman A, Villasboas M, et al. Use of colistin in a pediatric burn unit in Argentina. J Burn Care Research 2009;30(4):612-5.

25. Goverman J, Weber J, Keaney T, Sheridan R. Intravenous colistin for the treatment of multi-drug resistant, gramnegative infection in the pediatric burn population. J Burn Care Res 2007;28(3):421-6.

26. Gomes D, Macieira L Jr., Serra MC, Schechtmann M. Moderno tratamiento tópico de las quemaduras y utilización de antibioticoterapia sistémica. Rev Argent Quemad 2000;15(2):24-36.

27. ErolS, Altoparlak U, Akcay MN, Celebi F, Parlak M. Changes of microbial flora and wound colonization in burned patients. Burns 2004;30(4):357-61.

28. Hughes MG, Sawyer RW. Fungal infections in trauma and burn patients. Curr Treat Op Infect Dis 2001;3:499-505.

29. Church D, Elsayed S, Reid O, Winston B, Lindsay R. Burn wound infections. Clin Microbiol Rev 2006;19(2):403-34.

30. Belgian Outcome in Burn Injury Study Group. Development and validation of model for prediction of mortality in patients with acute burn injury. Br J Surg 2009;96(1):111-7 
308 / Arch Argent Pediatr 2013;111(4):303-308 / Original article

\section{ELECTRONIC ANEX}

TABLE 2. Sources of infection and etiologic agents ( $n$ : 128 sources in 84 patients)

\begin{tabular}{lcc} 
Source & $\mathbf{n}(\mathbf{\%})$ & Microorganismganism \\
\hline Sepsis associated to burn & $48(37)$ & Gram-negative bacilli \\
wound & MDR Pseudomonas aeruginosa \\
& MDR Acinetobacter sp. \\
& Stenotrophomonas maltophilia \\
& Enterobacter aglomerans \\
& Escherichia coli \\
& Serratia \\
& Klebsiella pneumoniae \\
Burkhordelia
\end{tabular}

$n$

20

4

3

3

2

2

1

1

Gram-positive

MR Stahylococcus aureus 8

MS Stahylococcus aureus 5

Enterococcus faecium $\quad 6$

Bacillus spp. 1

Nocardia

Fungi

Candida albicans

Non-typified filamentous

Non-typified no albicans

Candida guillermondie

Trichosporum asahi

Candida tropicalis

Candida lusitanae

Candida glabrata

Mucor

29 (23)

Burn wound

Gram-negative bacilli

MDR Pseudomonas aeruginosa

MDR Acinetobacter sp.

Klebsiella sp.

Enterobacter aglomerans

Alcaligenes xiloxosidans

Gram-positive

MR Stahylococcus aureus $\quad 4$

MS Stahylococcus aureus 2

Bacillus sp. 3

Corynebacterium sp. $\quad 2$

Enterococcus sp. 1

S. pyogenes

Fungi

Fusarium

Aspergillus fumigates

Alternaria sp.

Candida albicans

Candida tropicalis

Aureobasidium pullulans

Dreschelera

Non-typified filamentous

Penicilium

Mucor

Rodhotorula
5

4

3

2

2

1

1

1

9

5

2

1

5

5

3

1

3

1

1

1

1 
Line or catheter-associated infection

Urinary tract infection

associated with urinary catheter

MV-associated pneumonia

Osteomyelitis

\section{Pneumonia}

Corneal abscesses

Chondritis

Toxic shock

Probable endocarditis

$1(1)$

$1(1)$
Gram-negative bacilli

MDR Pseudomonas aeruginosa

MDR Acinetobacter sp.

Klebsiella pneumoniae

Serratia marcescens

Alcaligenes xylosoxidans

Gram-positive

MS S. aureus

Enterococcus faecium

Bacillus sp.

Fungi

Candida albicans

Candida parapsilopsis

Candida no albicans

Gram-negative bacilli

MDR Pseudomonas aeruginosa $\quad 7$

Enterobacter aglomerans $\quad 2$

Klebsiella pneumoniae $\quad 1$

MDR Acinetobacter spp. $\quad 1$

Fungi

Candida albicans

Candida tropicalis

Gram-negative bacilli

MDR Pseudomonas aeruginosa 6

MDR Acinetobacter sp. $\quad 2$

Klebsiella pneumoniae $\quad 1$

Alcaligenes xylosoxidans $\quad 1$

Positive cocci

MDR S. aureus

Fungi

Candida albicans

MDR S. aureus

Enteroccoccus faecalis

Streptococcus pneumoniae $\quad 1$

Negative cultures 3

MDR Pseudomonas aeruginosa 3

MDR Pseudomonas aeruginosa 2

Streptococcus pyogenes 1

MDR Acinetobacter spp. $\quad 1$

Varicella-zoster virus $\quad 1$

MR: multi-drug resistant; MR: methicillin-resistant; MS: methicillin-sensitive. 
310 / Arch Argent Pediatr 2013;111(4):303-308 / Original article

TABLE 3. Bivariate analysis ( $n$ : 110 patients)

\begin{tabular}{lccccc}
\hline Outcome measure & Infected n (\%) & Non-infected n (\%) & Total n (\%) & RR (95\% CI) & $p$ \\
\hline Type B burn & $47(43)$ & $5(4)$ & $52(47)$ & $1.42(1.14-0.75)$ & $<0.001$ \\
Percentage of burned body surface area & Md 34\% & Md 19\% & & Difference CI & $<0.0001$ \\
& & & & $(5-25 \%)$ & \\
Systemic antibiotic prophylaxis & $79(72)$ & $13(12)$ & $92(84)$ & $3.09(1.46-6.54)$ & $<0.0001$ \\
Topical antibiotics & $78(71)$ & $17(15)$ & $95(86)$ & $2.05(1.10-3.84)$ & $<0.01$ \\
Venous line & $77(70)$ & $13(12)$ & $90(82)$ & $2.44(1.34-4.47)$ & $<0.0001$ \\
Arterial line & $72(65)$ & $11(10)$ & $83(75)$ & $1.95(1.27-3)$ & $<0.0001$ \\
Urinary catheter & $73(66)$ & $13(12)$ & $86(78)$ & $1.85(1.19-0.89)$ & $<0.0001$ \\
Mechanical ventilation & $64(58)$ & $11(10)$ & $75(68)$ & $1.49(1.10-0.02)$ & $<0.01$ \\
Escharotomy & $77(70)$ & $13(12)$ & $90(82)$ & $2.44(1.34-0.47)$ & 0.0001 \\
Graft requirement & $71(64.5)$ & $11(10)$ & $82(74.5)$ & $2.05(1.33-0.17)$ & 0.0001 \\
Balneotherapy & $78(71)$ & $15(14)$ & $93(85)$ & $5.92(2.43-4.46)$ & 0.0001 \\
\hline
\end{tabular}

TABLE 4. Multivariate analysis of infection risk

\begin{tabular}{lccc}
\hline Outcome measure & RR & $\mathbf{9 5 \% ~ C I ~}$ & $p$ \\
\hline Central venous line & 5.15 & $1.44-18.46$ & 0.012 \\
Antibiotic prophylaxis & 5.22 & $1.26-21.63$ & 0.023 \\
Graft requirement & 3.65 & $1.08-12.37$ & 0.038 \\
\hline
\end{tabular}

